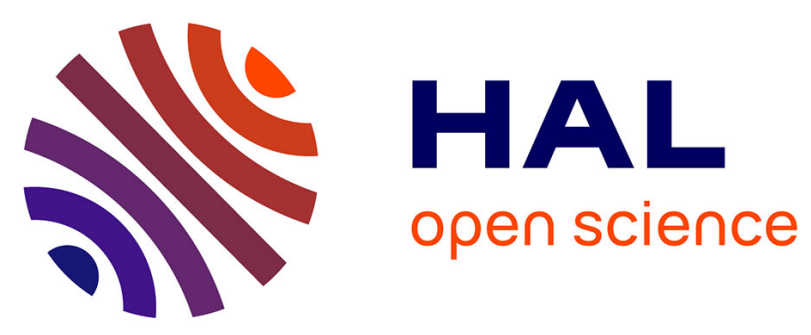

\title{
Mixed experimental and numerical approach for characterizing the biomechanical response of the human leg under elastic compression.
}

Stéphane Avril, Laura Bouten, Laura Dubuis, Sylvain Drapier, Jean-François Pouget

\section{To cite this version:}

Stéphane Avril, Laura Bouten, Laura Dubuis, Sylvain Drapier, Jean-François Pouget. Mixed experimental and numerical approach for characterizing the biomechanical response of the human leg under elastic compression.. Journal of Biomechanical Engineering, 2010, 132 (3), pp.031006. 10.1115/1.4000967 . emse-00509135

\section{HAL Id: emse-00509135 \\ https://hal-emse.ccsd.cnrs.fr/emse-00509135}

Submitted on 18 Jan 2011

HAL is a multi-disciplinary open access archive for the deposit and dissemination of scientific research documents, whether they are published or not. The documents may come from teaching and research institutions in France or abroad, or from public or private research centers.
L'archive ouverte pluridisciplinaire HAL, est destinée au dépôt et à la diffusion de documents scientifiques de niveau recherche, publiés ou non, émanant des établissements d'enseignement et de recherche français ou étrangers, des laboratoires publics ou privés. 


\title{
Mixed experimental and numerical approach for characterizing the biomechanical response of the human leg under elastic compression
}

\author{
Stéphane Avril · Laura Bouten · Laura \\ Dubuis · Sylvain Drapier • Jean-François \\ Pouget
}

Received: date / Accepted: date

\begin{abstract}
Elastic compression is the process of applying an elastic garment around the leg, supposedly for enhancing the venous flow. However, the response of internal tissues to the external pressure is still partially unknown. In order to improve the scientific knowledge about this topic, a slice of a human leg wearing an elastic garment is modelled by the finite-element method. The elastic properties of the tissues inside the leg are identified thanks to a dedicated approach based on image processing. After calibrating the model with MRI scans of a volunteer, the pressure transmitted through the internal tissues of the leg is computed. Discrepancies of more than $35 \%$ are found from one location to another, showing that the same compression garment cannot be applied for treating deficiencies of the deep venous system or deficiencies of the large superficial veins. Moreover, it is shown that the internal morphology of the human leg plays an important role. Accordingly, the approach presented in this paper may provide useful information for adapting compression garments to the specificity of each patient.
\end{abstract}

Keywords soft tissues · elastic compression · image warping · mechanical identification $\cdot$ FE modelling

\author{
Stéphane Avril · Laura Bouten · Laura Dubuis \\ Center for Health Engineering \\ Ecole Nationale Supérieure des Mines \\ PECM - CNRS UMR 5146 ; IFRESIS - INSERM IFR 143 \\ 158 Cours Fauriel, 42023 SAINT-ÉTIENNE cedex 2, FRANCE E-mail: avril@emse.fr \\ Sylvain Drapier \\ Structures and Materials Sciences Division \\ Ecole Nationale Supérieure des Mines \\ LTDS - CNRS UMR 5513 \\ 158 Cours Fauriel, 42023 SAINT-ÉTIENNE cedex 2, FRANCE \\ Jean-François Pouget \\ BVsport \\ 104, rue Bergson, 42000 SAINT-ÉTIENNE, FRANCE
}




\section{Introduction}

The major function of veins is to return blood back to the heart. In the leg, when veins fail to work properly, the classical therapy is elastic compression (EC): an elastic garment is applied around the leg. It is supposed to help the venous return, to decrease venous pressure, to prevent venous stasis and deterioration of venous walls, and to efficiently relieve aching and heavy legs [1].

Classically, the compression is prominent at the ankle and decreases going up the leg. This principle was established in reference to the Poiseuille's law in fluid mechanics which states that steady flow in a cylindrical pipe is always proportional to the opposite of pressure gradients. However, applying this principle to EC is arguable. Indeed, strong pressures at the ankle only affect the venous flow very superficially as ankles are mostly bony. Pressures at the ankle may even be painful and hamper the garment to be slipped on. Conversely, large pressures onto the calf muscle are essential, as confirmed by clinical studies [2] or medical studies based on Magnetic Resonance Imaging (MRI) and ultrasounds [3-6].

From a biomechanical point of view, no numerical model has ever been developed for reproducing the action of compression garments on legs and henceforward for helping to their design. Studies regarding the biomechanical effect of compression stockings on legs are limited to fluid mechanics $[7,8]$. They have never addressed the essential question of pressure transmission through the leg tissues.

The finite-element (FE) method can give a proper response to this mechanical issue. However, FE models considering the soft tissues of the human leg are quite scarce. Studies about the heel pad [9], the thigh tissues of amputees $[10,11]$ or the buttock [12] can be cited. However, the relevancy of such models is always conditioned by the accuracy of constitutive properties. Such properties are still very difficult to identify in vivo, even using recent techniques such as elastography [13-15]. 
The aim of this paper is to set up a patient-specific FE model of the human leg under the action of compression garments. For solving the issue of identifying the elastic properties for the soft tissues, a dedicated approach based on image processing has been developed. After detailing the FE model and the identification approach, results obtained on a volunteer are presented.

\section{Materials and methods}

2.1 Subject and images

\subsubsection{Image acquisition}

MRI scans of the leg cross section are necessary for this study. The scans were acquired with a $1.5 \mathrm{~T}$ MRI scanner on a volunteer, a 25-year-old woman without venous pathology, following informed consent and according to a protocol approved by the local institutional ethics committee. Scans were acquired in the natural state (without compression) of the leg, as shown in Fig. 1(a), but also while wearing compressive socks, as shown for example in Fig. 1(b). T1-weighted images of $5 \mathrm{~mm}$ thick slices were acquired under a spin echo sequence with a repetition time (TR) of $772 \mathrm{~ms}$ and an echo time (TE) of $13 \mathrm{~ms}$ [16], with a pixel size of about $0.36 \times 0.36 \mathrm{~mm}^{2}$. The cross section shown in Fig. 1 is located where the perimeter of the calf is the largest. It was segmented for defining the contours of the tibia and fibula bones, the adipose tissue (fat) and the muscle tissue. A T1-weighted sequence was employed here because it provides a good contrast between these tissues. Segmentation was achieved using the Matlab ${ }^{\circledR}$ software [17] with a custom made routine.

\subsubsection{Image warping}

In order to identify the material properties of soft tissues, an image processing approach based on image warping (also called image registration) was used [18]. The principle of image warping is the following. Let $I_{1}$ be the image to be warped, in our case 
the image of the leg wearing compressive socks (Fig. 1(b)). Let $I$ denote the warped image, i.e. a synthetic image derived from $I_{1}$ by mathematical operations. Actually, the mathematical operations consists here in applying onto $I_{1}$ a geometric deformation. The principle is the following:

1. Let $u(x, y)$ and $v(x, y)$ be respectively the horizontal and vertical components of the geometric deformation; $x$ and $y$ denote the plane coordinates of a pixel of the warped image where the grey level has to be estimated. Let $i(x, y)$ denote this gray level.

2. Assuming that the grey level of each material point remains constant between the unloaded and loaded states, it can be stated that $i(x, y)$ should be the same as $i_{1}(x+u, y+v), i_{1}(x+u, y+v)$ denoting the grey level at $(x+u, y+v)$ in $I_{1}$.

3. As $(x+u, y+v)$ is usually not the exact position of a pixel in $I_{1}, i(x, y)$ is derived by linear interpolation from the grey levels of the pixels surrounding $(x+u, y+v)$ in $I_{1}$.

Eventually, the objective is that $I$ matches as much as possible the target image, denoted $I_{0}$, which is the image of the uncompressed leg in our case. Image warping was achieved using the Matlab ${ }^{\circledR}$ software [17] with a custom made routine.

\subsection{Computational modeling}

A FE model of a leg was developed in this study. FE modelling consists in a number of components:

1. computational mesh generation,

2. specification of boundary conditions,

3. specification of material properties,

4. calibration of the material properties using experimental data (this step is only mandatory when the material properties are unknown),

5. simulation and results 
FE analyses were run on a software called Zebulon ${ }^{\circledR}$ [19]. Large deformations were considered in the resolution by taking into account geometric nonlinearities. The model was $2 \mathrm{D}$, representing a slice of the leg. Accordingly, the plane strain assumption was considered.

\subsubsection{Mesh generation}

The geometry of the internal tissues constituting the leg was deduced from the MRI scans of the leg cross section. The whole geometry was meshed with 400 displacementbased linear triangular elements (Fig. 2), which appeared to be satisfactory for the soft tissues considered here [20]. The mesh was created directly after the image segmentation, still using the Matlab ${ }^{\circledR}$ software [17] with a custom made routine. Then, the mesh was exported to Zebulon ${ }^{\circledR}$.

\subsubsection{Boundary conditions}

Boundary conditions were of two types:

- Dirichlet, by fixing all the degrees of freedom along the contours of the tibia and fibula bones, as both bones are assumed to be infinitely rigid (their elastic properties are nearly 1000 times larger than the ones of soft tissues).

- Neumann along the external contour of the leg. A normal pressure was applied along this contour. Locally, the pressure value, denoted $p$, is computed as a function of the local tension in the garment, denoted $T$ (defined as the force per unit width), and the local radius of curvature, denoted $r$ (evaluated in the deformed configuration of the leg during the FE computations), following the Laplace's law:

$$
p=T / r
$$

Friction of the garment on the leg is neglected, which means that no tangential pressure is applied. Consequently, for simple mechanical balance arguments, the $T$ 
value is assumed as constant along the contour of the leg. Thanks to the segmentation of the MRI scan of the leg before and after wearing the compression garment, it is possible to evaluate the perimeter of the leg in both uncompressed and compressed states. Then the stretch of the garment in the circumferential direction can be deduced. Thus, the $T$ value in Eq. 1 is deduced by characterizing previously the elastic response of the garment.

\subsubsection{Garment characterization}

A non destructive system, shown in Fig. 3, has been devised for the mechanical characterization, reproducing loading conditions similar to the ones observed in the circumferential direction of the leg when the garment is worn. This system and the related tests result from an original methodology for characterizing the biaxial behaviour of elastomeric textiles $[21,22]$. This methodology is based on a specific experimental set-up for applying biaxial loadings to the garment, and on an optical system for measuring the deformation of the garment during its loading [22]. Thanks to this system, the value of $T$ was estimated with an accuracy of about $10 \%$. A complementary study was also carried out for verifying the relevancy of the Laplace's law [21].

\subsubsection{Material properties}

Image segmentation has yielded different zones in the images: one corresponding to the muscle tissues, another one corresponding to adipose tissues, and the others being the bones. In the model, the zones of the muscle and adipose tissues were assumed as homogeneous in terms of elastic properties. Each tissue is figured by a different colour in Fig. 2. The skin, the veins and the arteries are not modelled. The skin is included in the fat zone. Similarly, veins and arteries are included in the muscle or adipose tissue. In terms of elastic properties, it is assumed that the veins and arteries contribute to the average elastic behaviour of the tissue where they sit (fat or muscle). The assumption 
of homogeneity in both tissues provides a sufficient accuracy for evaluating the strains and stresses inside the leg, as this will be shown in Section 4.2.

The response of the soft tissues of the leg is assumed as compressible hyperelastic. Compressibility is justified by observing a diminution of about $4.5 \%$ of areas in the MRI scans when comparing scans before and after wearing the garment (Fig. 1). As the finite deformations of the soft tissues remain relatively small $(<5 \%)$, a simple NeoHookean strain energy function is justified [23]. This strain energy function can be written:

$$
W=C_{10}\left(I_{1}-3\right)+K_{v}\left[\left(J_{2}-1\right) / 2-\ln (J)\right]
$$

where $J=\operatorname{det}(F)$ and $I_{1}=J-2 \operatorname{tr}\left({ }^{t} F . F\right) / 3$ with $F$ being the deformation gradient and tr the trace of a matrix.

The elastic behaviour of each tissue is driven by two parameters: $C_{10}^{m}$ and $K_{v}^{m}$ for the muscle tissue and $C_{10}^{f}$ and $K_{v}^{f}$ for the fat. The model is therefore driven by 4 parameters. There is a very large variability of the values reported for the elastic parameters of soft tissues in the literature $[10-12,24]$. One objective of this paper is to propose a methodology for calibrating the 4 parameters as realistically as possible with regard to the in vivo response of any patient's leg.

\subsubsection{Calibration against experimental data}

After checking convergence of the FE model on representative materials, the problem of identifying $C_{10}^{m}, K_{v}^{m}, C_{10}^{f}$ and $K_{v}^{f}$ from real experimental data was addressed. Using the MRI scans as experimental data, this entailed using image processing algorithms such as digital image correlation (DIC) [25], or image warping (also called image registration) [18]. DIC proved not to be suited to such in vivo identification [20]. On the contrary, the use of image registration applied to medical images for identifying the elastic properties of soft tissues was already used in different studies [12,26-29]. 
Let $I_{0}$ be the image of the leg in the unloaded state (Fig. 1(a)), and let $I_{1}$ be the image of the leg in the loaded state (Fig. 1(b)). Let $I$ be the processed image deduced from $I_{1}$ after warping, by applying the deformation computed by the FE model, as explained in section 2.1.2. Therefore, the warped image $I$ only depends on $C_{10}^{m}, K_{v}^{m}$, $C_{10}^{f}$ and $K_{v}^{f}$ as these four parameters drive the response computed by the FE model. Let $\mathcal{M}$ be the measure of mismatching between the original image $I_{0}$ and the warped image $I . \mathcal{M}$ only depends on the unknown constitutive parameters and it can be denoted as a function of them: $\mathcal{M}\left(C_{10}^{m}, K_{v}^{m}, C_{10}^{f}, K_{v}^{f}\right)$. The measure of mismatch can be of different types, defined for example on the basis of cross correlation products, or otherwise by the distance separating the same markers on both images. This second option has been chosen here, using the contours of the fat and muscle zones as markers (Fig. 1). This option has been chosen instead of using correlation products because, for the correlation products, the contrast of MRI images is too poor.

For each pixel of the contours in $I$, of coordinates $\left(x_{k}, y_{k}\right)$, the closest pixel belonging to a contour of fat or muscles in $I_{0}$ is found, and the distance between them is denoted $d_{k}$. Graphical plots of $d_{k}$ 's are shown in Fig. 4(a) and in Fig. 4(b). This type of graphical plots shows where the mismatch is the largest, which can help to interpret the relevancy of the FE model. Eventually, the measure of mismatching $\mathcal{M}\left(C_{10}^{m}, K_{v}^{m}, C_{10}^{f}, K_{v}^{f}\right)$ is defined as the sum of all the $\left(d_{k}\right)^{2}$ 's for all the pixels belonging to a contour.

The optimal registration is reached when the four parameters $C_{10}^{m}, K_{v}^{m}, C_{10}^{f}$ and $K_{v}^{f}$ minimize $\mathcal{M}\left(C_{10}^{m}, K_{v}^{m}, C_{10}^{f}, K_{v}^{f}\right)$. Such minimization is achieved by running the Nelder Mead algorithm in the Matlab ${ }^{\circledR}$ software [17]. In this algorithm, evaluations of the cost function $\mathcal{M}\left(C_{10}^{m}, K_{v}^{m}, C_{10}^{f}, K_{v}^{f}\right)$ are required through an iterative scheme. For each evaluation of the cost function here, a new FE analysis is required, with new inputs of the elastic properties of the muscle and adipose tissues. In the Matlab ${ }^{\circledR}$ software, a routine was developed for running these FE analyses, saving the results 
(displacement fields $u$ and $v$ ), and using them for achieving image registration and update $\mathcal{M}\left(C_{10}^{m}, K_{v}^{m}, C_{10}^{f}, K_{v}^{f}\right)$.

Dialogue between Matlab ${ }^{\circledR}$ and Zebulon ${ }^{\circledR}$ was achieved by including prompt commands in the Matlab ${ }^{\circledR}$ routines that order the jobs to the Zebulon ${ }^{\circledR}$ software. Before each new job, the data file containing the material properties of the FE model required by Zebulon ${ }^{\circledR}$ was updated with the new values of $C_{10}^{m}, K_{v}^{m}, C_{10}^{f}$ and $K_{v}^{f}$ for which a new evaluation of the cost function is needed.

\subsubsection{Statistical approach}

An alternative approach has been used to determine mechanical properties. This alternative approach does not provide a unique value for the material properties but it provides intervals within which the material properties are likely to be. Instead of minimizing $\mathcal{M}\left(C_{10}^{m}, K_{v}^{m}, C_{10}^{f}, K_{v}^{f}\right)$ by running the Nelder Mead algorithm, a Monte Carlo method is used [30]. Material properties are drawn randomly in intervals of consistent values, and for each set of random $C_{10}^{m}, K_{v}^{m}, C_{10}^{f}$ and $K_{v}^{f}$, the value of $\mathcal{M}\left(C_{10}^{m}, K_{v}^{m}, C_{10}^{f}, K_{v}^{f}\right)$ is computed and the material properties leading to a value of $\mathcal{M}$ lower than a given threshold are kept.

\section{Results}

3.1 Elastic properties of tissues

The elastic properties of the soft tissues were identified by applying the approach described in section 2.2.5. It was observed that, whatever the values of $C_{10}^{m}, K_{v}^{m}, C_{10}^{f}$ and $K_{v}^{f}$ chosen within a broad range (Tab. 1) for initializing the iterative scheme, the minimization always converged, though yielding different results. This means that the cost function has several minima.

Accordingly, it was decided to use another approach for the minimization. It was observed that when the value of the cost function passed below 3.4, the variations of the cost function with respect to the values of $C_{10}^{m}, K_{v}^{m}, C_{10}^{f}$ and $K_{v}^{f}$ become very 
irregular and they can not be controlled. Therefore, all the values of $C_{10}^{m}, K_{v}^{m}, C_{10}^{f}$ and $K_{v}^{f}$ that provide cost function values below 3.4 are considered as satisfactory. It is shown in Section 4.2 that the nonuniqueness of the solution has only a limited impact on the uncertainty of pressure computations.

Using the statistical approach presented in section 2.2.5, it was found that the values of $C_{10}^{m}, K_{v}^{m}, C_{10}^{f}$ and $K_{v}^{f}$ that provide cost function values below 3.4 are located in intervals delimited by an upper bound and a lower bound. Out of these bounds, the material parameters yield too large values of the cost function. The bounds of these intervals along with the central values are reported in Tab. 2.

3.2 Pressure evaluation with central values of elastic properties

As the veins have a small size compared with the size of the fat and muscle areas in the scans (Fig. 1), it can be assumed that the presence of a vein in the fat or muscle tissues does not drastically modify the local stresses computed by our model. Therefore, the pressure that tissues will apply onto the vein walls can be approximated by the local hydrostatic pressure (first invariant of the stress tensor) evaluated in the fat or muscle tissues by our model.

The obtained distribution of pressure is shown in Fig. 5, computed with the central values of elastic properties reported in Tab. 2. A special attention has been paid to the results at five positions (Fig. 5) which may have an interest for physicians (results for these five locations are reported in the first column of Tab. 3):

- position 1 is located in the hypodermis, close to the tibia bone.

- position 2 is located in the deep venous system near the twin veins.

- position 3 is located where the maximum pressure is usually observed by our FE model.

- position 4 is located near the greater saphenous, which is the large superficial vein on the inner side of the human leg. 
- position 5 is located near the small saphenous vein, which is a large vein of the superficial posterior leg.

The maximum pressure computed is of $5.3 \mathrm{kPa}(40.3 \mathrm{mmHg})$, it is obtained at the back of the leg, at the interface between the fat and the muscle tissues. The minimum pressure is of $0.9 \mathrm{kPa}(6.8 \mathrm{mmHg})$, it is obtained near the tibia bone. The first important conclusion is that the spatial variations of pressures are significant and consequently, it cannot be assumed that the same pressure is applied onto the whole veins. The variation between the pressures computed at position 2 and position 3 is more than $35 \%$.

\section{Discussion}

\subsection{Material properties}

The bounds reported in Tab. 2 for $C_{10}^{m}, K_{v}^{m}, C_{10}^{f}$ and $K_{v}^{f}$ are in agreement with the range order of values known for the properties of soft tissues like fat or muscles $[10-12,24]$. The fat properties found here are also similar to hypodermis elastic properties reported in the literature $[31,32]$. This supports the simplification of merging the adipose tissues and the skin in the present model.

4.2 Irregularity of the cost function and nonuniqueness of the solution of the inverse problem

It was observed that when the value of the cost function passed below 3.4, the variations of the cost function with respect to the values of $C_{10}^{m}, K_{v}^{m}, C_{10}^{f}$ and $K_{v}^{f}$ become very irregular and they can not be controlled. The irregularity of the cost function can be understood by observing the local mismatch between the warped image and the target image. Indeed, using values of $C_{10}^{m}, K_{v}^{m}, C_{10}^{f}$ and $K_{v}^{f}$ located within the bounds reported in Tab. 2, it was noticed that when the local mismatch is minimized on one part of the contours, it is always increased elsewhere. For example, Fig. 4(a) 
and Fig. 4(b), obtained with two different sets of elastic properties, show some minor registration errors located at different locations, whereas they both correspond to cost function values located below 3.4.

Accordingly, there is not a unique set of material parameters ensuring a good agreement between the numerical and actual deformations of the leg. The consequence for the current approach is that the values of the pressures inside the leg cannot be computed deterministically. Pressures reported in the first column of Tab. 3 are mean values, and it can only be announced that the actual pressures may be scattered about these mean values.

In order to evaluate this scatter about the mean values, 30 computations were achieved with the FE model, each one using a set of elastic properties drawn randomly within the bounds reported in Tab. 2. This approach for characterizing uncertainty has the advantage of being statistically relevant and not very time consuming (15 minutes in total).

The coefficients of variation (ratio between the standard deviation and the mean value) of the 30 pressure values were systematically smaller than $5 \%$ (third column in Tab. 3). Therefore, this shows that the nonuniqueness of the identified material parameters has only a limited impact on the uncertainty of pressure computations.

\subsection{Image processing}

In this study, the contours of the fat and muscle zones were used as markers (Fig. 1(a)) instead of using correlation products because, for the correlation products, it must be assumed that the MRI signal received from each material point of the leg is constant between the unloaded and loaded states, and also because the contrast inside the fat or muscle tissues is poor. Different MRI modalities like MRI tagging may help to provide a better contrast inside the tissue. This would increase the number of markers and consequently improve the sensitivity of the cost function to the material properties. 
4.4 Effect of the leg morphology

In our approach, the contours of the fat and the muscle tissues were deduced from the MRI scans. A T1-weighted sequence was employed because it provides a good contrast between these tissues. A question arises from this segmentation: is there also a contrast between these two zones in terms of mechanical properties?

Let us consider that the central value of intervals found for each property is their most probable guess. Therefore, regarding these values, the muscle tissue is significantly more compressible than fat. This is also in agreement with the variation of their respective area between Fig. 1(a) and Fig. 1(b). This means that EC has a larger effect on the muscle zone than on the fat zone. Therefore, it transpires that the proportion of fat inside the leg can play an important role in the efficiency of the compression process. This analysis is in agreement with several medical studies about EC, which showed that most of the venous blood in the leg is located in venules of the calf muscle [4-6].

The contrast of mechanical properties also induces a contrast of computed pressures. There is indeed a slight jump of pressure at the interface between the fat and the muscle tissues (Fig. 5), due to the difference of elastic properties. This effect shows that the fat has a "shielding" effect, lowering the pressures inside muscles. Therefore, this confirms that the internal leg morphology should be considered before the prescription of EC.

4.5 Uncertainty about the boundary conditions

A large source of uncertainty about pressure evaluation is induced by the uncertainty of the garment tension. Experimentally, tension $T$ can only be assessed with a precision of about $10 \%$. This includes the fact that the friction is neglected in the boundary conditions.

The increase or decrease of computed pressures due to a respective increase or decrease of tension is not linear, as increases or decreases range from 5 to $14 \%$ (Tab. 4). 
The nonlinearity is induced by the geometric nonlinearity of the FE model, especially by the fact that the Laplace's law is calculated with the deformed value of the local radius of curvature $r$. However, a rough estimate of about $10 \%$ can still be kept for characterizing the uncertainty of pressure evaluations induced by the uncertainty of tension evaluation.

Eventually, adding up the uncertainty of the elastic properties to the uncertainty of the garment tension, it can be concluded that the pressure inside the leg induced by EC can be assessed by our approach with a relative precision of about $15 \%$. For classically prescribed pressures of about $20 \mathrm{mmHg}$, this corresponds to an uncertainty of $3 \mathrm{mmHg}$, which is a common tolerance for such medical prescriptions.

A natural improvement would be to include the garment in the numerical model, with contact elements between the garment and the leg. The question that arises is whether the time for running such model will be balanced by a worthwhile increase in accuracy. This question will be addressed in the near future.

4.6 Link with the blood pressure

An assumption is made to obtain the pressure applied onto the vein walls. The mechanical role of veins is neglected and the pressure is computed as the hydrostatic pressure in the surrounding tissues. It is a strong assumption, the limitations of which are not evaluated in the current study. A more sophisticated model involving fluid structure interactions will be set up in the future for addressing this issue. In the meantime, the current study still provides an answer to the general question of pressure transmission through the internal tissues. The current model shows that the spatial variations of pressures are significant inside the leg and consequently, pressure transmission through the internal tissues should be considered in medical treatments.

Avril - BIO-09-1119 
$4.73 \mathrm{D}$ effects

A strong assumption in our approach is the plane strain assumption. It means that the out of plane strains are zero. In solid mechanics, the plane strain assumption is employed when the dimension of a structure in one direction is very large in comparison with the dimensions of the structure in the two other directions. The leg can be assumed as such a structure.

In order to justify this assumption, a 3D FE model was set up. The geometry was deduced from the scans of all the cross sections of the whole leg using the Avizo $(\mathbb{R}$ software [33]. The geometry was meshed with 718,937 tetraedric linear elements with 131,712 nodes. The material properties and the boundary conditions were similar to the ones used in 2D: the bones were clamped and pressure was applied onto the skin. The mesh and the boundary conditions are shown in Fig. 6(a). Using this model, the deformation of the leg tissues was computed, as shown in Fig. 6(b).

Eventually, a comparison was made between the pressures at the five positions of Fig. 5 computed with the $2 \mathrm{D}$ or with the $3 \mathrm{D}$ model. The results are reported in Tab. 5 . It can be checked that the difference between the pressures of the $2 \mathrm{D}$ model and of the $3 \mathrm{D}$ model is low, less than $5 \%$, which remains within the range of uncertainty of the approach reported before.

It must be emphasized that the computation speed with a $2 \mathrm{D}$ model is drastically reduced compared with a 3D model, especially with the material and geometric nonlinearities considered here. The main advantage of the $2 \mathrm{D}$ model presented here is that it can provide almost in real time the distribution of pressures inside the leg for any patient provided that the internal morphology of the leg is known (only 2 MRI scans are required). Regarding the uncertainties, the $2 \mathrm{D}$ model is able to predict the internal pressures with an uncertainty of $15 \%$, which is still compatible with the requirements of medical prescriptions. 
4.8 Number of patients

The results shown in this paper prove the feasibility of a FE model of the human leg under elastic compression where the material parameters of the FE model are identified from in vivo data. This was shown on a single subject without venous pathology. Such an approach should now be validated on different sets of subjects in order to take into account the wide variations of properties from one subject to another. The effect of venous pathologies onto the approach should also be investigated.

\section{Conclusion}

In order to improve the scientific knowledge about the mechanical response of a human leg under elastic compression, a FE model was developed, under the assumption of plane strains. A methodology for calibrating the model in a patient specific way has been presented and the uncertainty of the results provided by the model has been characterized.

Using the model, it has been shown that the spatial variations of pressures are significant inside the leg. Discrepancies of more than $35 \%$ were reported from one location to another, showing that the same compression garment cannot be applied for treating deficiencies of the deep venous system or deficiencies of the large superficial veins. Moreover, the internal morphology of the human leg (muscle properties, thickness of subcutaneous fat) affects the pressure transmission through the internal tissues of the leg. A clinical validation is now required on a significant number of patients.

In the meantime, a more realistic model will also be implemented for analyzing the effects of the computed pressures onto the venous return. A 3D model has been set up, considering a more accurate description of the leg components. Blood flow in the veins of the compressed leg will be considered, based on different approaches of the literature $[7,8]$. 
Moreover, it should be emphasized that in the model used here, the reduction of volume in the muscle zone is fully attributed to the compressibility of the muscle tissue. This is likely to be abusive and a biphasic model considering the calf muscle as a wet "sponge", made of "real" muscle tissues and dense blood capillaries, would be more realistic. Future improvements of our model will go into this direction.

The recent progresses of the spatial resolution in medical imaging techniques and/or the combination of different MRI sequences may be the key for improving the accuracy of our in vivo identification methodology based on image registration. Moreover, such identification from in vivo data may have a lot of other applications outside the study of EC, since the mechanical characterization of soft tissues remains one of the most complicated issues in biomechanics.

When all the on-going improvements and validations are completed, the approach presented in this paper may become a useful tool in practice for adapting compression garments to the specificity of each patient.

\section{Acknowledgement}

The authors are grateful to the Rhone-Alpes Regional Council for funding the PhD grant of Laura Dubuis. This work is also part of the Imandef project (Grant ANR-08JCJC-0071) funded by the ANR (French National Research Agency). 


\section{References}

1. G. Belcaro, D. Christopoulos, and A.N. Nicholaides. Hémodynamique veineuse des membres inférieurs. Annales de Chirurgie Vasculaire, 4:305-330, 1991.

2. P. Ducrozet. Un nouveau concept de contention-compression veineuse évalué par un essai randomisé en double aveugle: contention progressive versus contention classique dans l'insuffisance veineuse légère: l'étude booster. Thèse de médecine, Université de Saint Etienne (in French), 44, 2004.

3. P. Blanchemaison, P. Gorny, and G. Louis. Relation entre veines et muscles du mollet chez le sportif et le sujet sédentaire : étude échographique et conséquences physiopathologiques. Phlébologie, 5:435-440, 1995.

4. J.F. Pouget, W.M.W. Geedroyc, and M. Prüfer. Nuclear magnetic resonance (NMR) study of leg venous drainage in men with and without elastic compression socks (traditional and new BVSport $\left.{ }^{(}\right)$concept). International Angiology, 19, 2000.

5. S. Couzan, M. Prüfer, and J.M. Ferret. Un nouveau concept de contention-compression : apport de l'écho-doppler couleur avec prise des pressions veineuses et de l'IRM. Phlébologie, 55:159-171, 2002.

6. S. Couzan, J.F. Pouget, M. Prüfer, J.M. Ferret, and P. Mismetti. Study of the athletes venous system by doppler scan with pressures measurement and the perfecting of a new elastic compression concept. International Angiology, 19 (suppl.1 to No 2), 2000.

7. B. Maton, G. Thiney, A. Ouchène, P. Flaud, and P. Barthelemy. Intramuscular pressure and surface EMG in voluntary ankle dorsal flexion: Influence of elastic compressive stockings. Journal of Electromyography and Kinesiology, 16:291-302, 2006.

8. S.P. Downie, S.M. Raynor, D.N. Firmin, N.B. Wood, S.A. Thom, A.D. Hughes, K.H. Parker, J.H.N. Wolfe, and X. Yun Xu. Effects of elastic compression stockings on wall shear stress in deep and superficial veins of the calf. American Physiological Society Heart and Circulatory Physiology, 294:H2112-H2120, 2008.

9. A. Gefen. Stress analysis of the standing foot following surgical plantar fascia disease. Journal of Biomechanics, 35:629-637, 2002.

10. S. Portnoy, Z. Yizhar, N. Shabshin, Y. Itzchak, A. Kristal, Y. Dotan-Marom, I. Siev-Ner, and A. Gefen. Internal mechanical conditions in the soft tissues of a residual limb of a trans-tibial amputee. Journal of Biomechanics, 41:1897-1909, 2008.

11. W.C.C. Lee and M. Zhang. Using computational simulation to aid in the prediction of socket fit: A preliminary study. Medical Engineering and Physics, 29:923-929, 2007.

12. E. Linder-Ganz, N. Shabshin, Y. Itzchak, and A. Gefen. Assessment of mechanical conditions in sub-dermal tissues during sitting: A combined experimental-MRI and finite element approach. Journal of Biomechanics, 40:1443-1454, 2007.

13. J. Ophir, F. Kallel, T. Varghese, E. Konofagou, S.K. Alama, T. Krouskop, B. Garra, and R. Righetti. Optical and acoustical imaging of biological media: Elastography. Comptes Rendus de l'Académie des Sciences Paris, 2, Série IV:1193-1212, 2001.

14. E. Park and A.M. Maniatty. Shear modulus reconstruction in dynamic elastography: time harmonic case. Physics in Medicine and Biology, 51:3697-3721, 2006.

15. P.E. Barbone and A.A. Oberai. Elastic modulus imaging: some exact solutions of the compressible elastography inverse problem. Physics in Medicine and Biology, 52:15771593, 2007. Institute of Physics Publishing.

16. D.W. MacRobbie, E.A. Moore, M.J. Graves, and M.R. Prince. MRI: From Picture to Proton. Cambridge University Press, 2003.

17. Matlab ${ }^{\circledR}$. http://www.mathworks.com, 2008.

18. K. Amunts, P.H. Weiss, H. Mohlberg, P. Pieperhoff, S. Eickhoff, J.M. Gurd, J.C. Mershall, N.J. Shah, G.R. Fink, and K. Zilles. Analysis of neural mechanisms underlying verbal fluency in cytoarchitectonically defined stereotaxic space - the role of Brodmann areas 44 and 45. NeuroImage, 22:42-56, 2004.

19. Zebulon $\mathbb{B}$. http://www.nwnumerics.com, 2008.

20. L. Bouten and S. Drapier. In-vivo identification of soft biological tissues using MR imaging. European Journal of Computational Mechanics, 18(1):21-32, 2009.

21. I. Gaied, S. Drapier, and B. Lun. Experimental assessment and analytical 2d predictions of the stocking pressures induced on a model leg by medical compression stockings. Journal of Biomechanics, 39:3017-3025, 2006.

22. I. Gaied and S. Drapier. Identification strategy for orthotropic knitted elastomeric fabrics under large biaxial deformations. Inverse Problems in Science and Engineering, 15(8):871$894,2008$. 
23. Y.C. Fung. Biomechanics: mechanical properties of living tissues. Springer, 1993.

24. A. Gefen, J. Chen, and D. Elad. A biomechanical model of peyronie's disease. Journal of Biomechanics, 33:1739-1744, 2000.

25. H. Schreier and M.A. Sutton. Systematic errors in digital image correlation due to undermatched subset shape functions. Experimental Mechanics, 42:303-310, 2002.

26. K.F. Augenstein, B.R. Cowan, I.J. Legrice, and A.A. Young. Estimation of cardiac hyperelastic material properties from MRI tissue tagging and diffusion tensor imaging. In MICCAI 2006, LNCS 4190, pages 628-635, 2006. Springer-Verlag Berlin Heidelberg.

27. M. Chabanas, Y. Payan, C. Marecaux, P. Swider, and F. Boutault. Comparison of linear and non-linear soft tissue models with post-operative CT scan in maxillofacial surgery. Lecture Notes in Computer Science, 3078/2004:19-27, 2006.

28. F.S. Azar, D.N. Metaxas, and M.D. Schnalli. Methods for modeling and predicting mechanical deformations of the breast under external perturbations. Medical Image Analysis, $6: 1-27,2002$.

29. J.H. Chung, V. Rajagopal, P.M.F. Nielsen, and M. P. Nash. A biomechanical model of mammographic compressions. Biomechanics and Modelling in Mechanobiology, 7:43-52, 2007.

30. K. Mosegaard and A. Tarantola. Monte carlo sampling of solutions to inverse problems. Journal of Geophysical Research, 100:12431-12447, 1995.

31. H. V. Tran, F. Charleux, A. Ehrlacher, and M-C. Ho Ba Tho. Propriétés mécaniques multi-couches de la peau humaine in vivo. In Colloque National en Calcul des Structures, 2005

32. A. Delalleau, G. Josse, J.M. Lagarde, H. Zahouani, and J.M. Bergheau. Characterization of the mechanical properties of skin by inverse analysis combined with the indentation test. Journal of Biomechanics, 39(9):1603-1610, 2005.

33. Avizo ${ }^{\circledR}$. http://www.vsg3d.com, 2009. 


\section{List of Tables}

1 Results obtained in the minimization process for different initial values. 21

2 Solution of the identification in the form of intervals. . . . . . . . . . . . 22

3 Pressure variations and uncertainty due to the approximation of tissue elastic properties. . . . . . . . . . . . . . . . . . 23

4 Pressure variations and uncertainty due to the approximation of the garment tension. . . . . . . . . . . . . . . . 24

5 Comparison of the pressures computed with the 2D and the 3D model. . 25 


\begin{tabular}{|c|c|c|c|c|c|c|c|c|}
\hline \multicolumn{3}{|c|}{$\begin{array}{c}\text { Initial values of elastic } \\
\text { properties }(\mathrm{kPa})\end{array}$} & $\begin{array}{c}\text { Mismatch value } \mathcal{M} \\
\text { after minimization }\end{array}$ & \multicolumn{4}{|c|}{$\begin{array}{c}\text { Elastic properties at } \\
\text { minimum }(\mathrm{kPa})\end{array}$} \\
\hline$C_{10}^{f}$ & $C_{10}^{m}$ & $K_{v}^{f}$ & $K_{v}^{m}$ & & $C_{10}^{f}$ & $C_{10}^{m}$ & $K_{v}^{f}$ & $K_{v}^{m}$ \\
\hline 7.92 & 10.1 & 86 & 69.5 & 3.36 & 7.2 & 9.9 & 88.5 & 74.1 \\
\hline 6.48 & 10.1 & 86 & 69.5 & 3.3 & 6.5 & 10.3 & 86.5 & 69.5 \\
\hline 7.2 & 9.1 & 86 & 69.5 & 3.22 & 3.3 & 11.5 & 89.6 & 71.9 \\
\hline 7.2 & 10.1 & 94.6 & 69.5 & 3.35 & 7.1 & 9.9 & 99.5 & 70.1 \\
\hline 7.2 & 10.1 & 77.4 & 69.5 & 3.32 & 7.3 & 10.1 & 80 & 69.6 \\
\hline 7.2 & 10.1 & 86 & 76.45 & 3.34 & 7.1 & 10 & 86.9 & 75.4 \\
\hline 7.2 & 10.1 & 86 & 62.55 & 3.31 & 6.2 & 10.4 & 91.3 & 69 \\
\hline 10 & 10 & 50 & 50 & 3.63 & 4.8 & 10.8 & 48.5 & 81.8 \\
\hline 7 & 10 & 35 & 40 & 3.75 & 3.4 & 9.7 & 49.4 & 74.6 \\
\hline 10 & 20 & 30 & 80 & 3.71 & 13.8 & 14.7 & 47.9 & 79.7 \\
\hline 9 & 13 & 70 & 80 & 3.62 & 8.7 & 13.1 & 74.1 & 78.6 \\
\hline 7 & 10 & 85 & 70 & 3.36 & 1.8 & 17 & 84.9 & 77.6 \\
\hline 7 & 10 & 85 & 70 & 3.33 & 7.2 & 10.1 & 86 & 69.5 \\
\hline
\end{tabular}

Table 1 Results obtained in the minimization process for different initial values. $C_{10}^{f}$ and $K_{v}^{f}$ are the material constants of fat, $C_{10}^{m}$ and $K_{v}^{m}$ are the material constants of the muscle tissue. $C_{10}$ is the constant corresponding to the contribution of the strain first invariant in the Neo-Hookean strain energy function, $K_{v}$ is the constant corresponding to the contribution of compressibility in the Neo-Hookean strain energy function. 


\begin{tabular}{|c|c|c|c|c|}
\hline & $C_{10}^{f}(\mathrm{kPa})$ & $C_{10}^{m}(\mathrm{kPa})$ & $K_{v}^{f}(\mathrm{kPa})$ & $K_{v}^{m}(\mathrm{kPa})$ \\
\hline lower bound & 2 & 9.4 & 71.3 & 61 \\
\hline upper bound & 7 & 12.9 & 140 & 78 \\
\hline central value & 4.5 & 11.2 & 106 & 69.5 \\
\hline
\end{tabular}

Table 2 Solution of the identification in the form of intervals. 


\begin{tabular}{|l|c|c|c|}
\hline & Average $(\bar{p})$ & Standard deviation & Coeff. of variation (\%) \\
\hline position 1 & 3.42 & 0.16 & 4.7 \\
\hline position 2 & 2.74 & 0.1 & 3.6 \\
\hline position 3 & 4.42 & 0.16 & 3.6 \\
\hline position 4 & 4.15 & 0.08 & 1.9 \\
\hline position 5 & 4.32 & 0.13 & 3 \\
\hline
\end{tabular}

Table 3 Pressure variations and uncertainty due to the approximation of tissue elastic properties. 


\begin{tabular}{|c|c|c|c|c|c|}
\hline & Average tension & \multicolumn{2}{|c|}{ Average tension $+10 \%$} & \multicolumn{2}{c|}{ Average tension $-10 \%$} \\
\cline { 2 - 6 } & Pressure $(\mathrm{kPa})$ & Pressure $(\mathrm{kPa})$ & Deviation $(\%)$ & Pressure $(\mathrm{kPa})$ & Deviation $(\%)$ \\
\hline position 1 & 3.4 & 3.77 & 11 & 2.94 & 14 \\
\hline position 2 & 2.72 & 2.95 & 8.4 & 2.46 & 9.5 \\
\hline position 3 & 4.38 & 4.66 & 6.4 & 4.13 & 5.7 \\
\hline position 4 & 4.15 & 4.48 & 8 & 3.74 & 10 \\
\hline position 5 & 4.28 & 4.71 & 10 & 3.83 & 10.5 \\
\hline
\end{tabular}

Table 4 Pressure variations and uncertainty due to the approximation of the garment tension. 


\begin{tabular}{|c|c|c|c|}
\hline & Pressure with the 2D model $(\mathrm{kPa})$ & Pressure with the 3D model (kPa) & Deviation \\
\hline position 1 & 3.42 & 3.39 & $1 \%$ \\
\hline position 2 & 2.74 & 2.81 & $3 \%$ \\
\hline position 3 & 4.42 & 4.4 & $0.5 \%$ \\
\hline position 4 & 4.15 & 3.99 & $4 \%$ \\
\hline position 5 & 4.32 & 4.5 & $5 \%$ \\
\hline
\end{tabular}

Table 5 Comparison of the pressures computed with the 2D and the 3D model. 


\section{List of Figures}

1 MRI scans of the leg with and without EC . . . . . . . . . . . 27

2 Mesh of the leg in the 2D FE model . . . . . . . . . . . . . . 28

3 Set-up used to characterize the elastic behaviour of compression garments 29

4 Graphical display of the mismatch between the target image and examples of registration. . . . . . . . . . . . . . . . . . 30

5 Hydrostatic pressure computed inside the leg with the central values of elastic properties reported in Tab. $2 \ldots \ldots$. . . . . . . . . 31

$6 \quad$ 3D FE model. . . . . . . . . . . . . . . . . . . . . . . . . . . . 32 


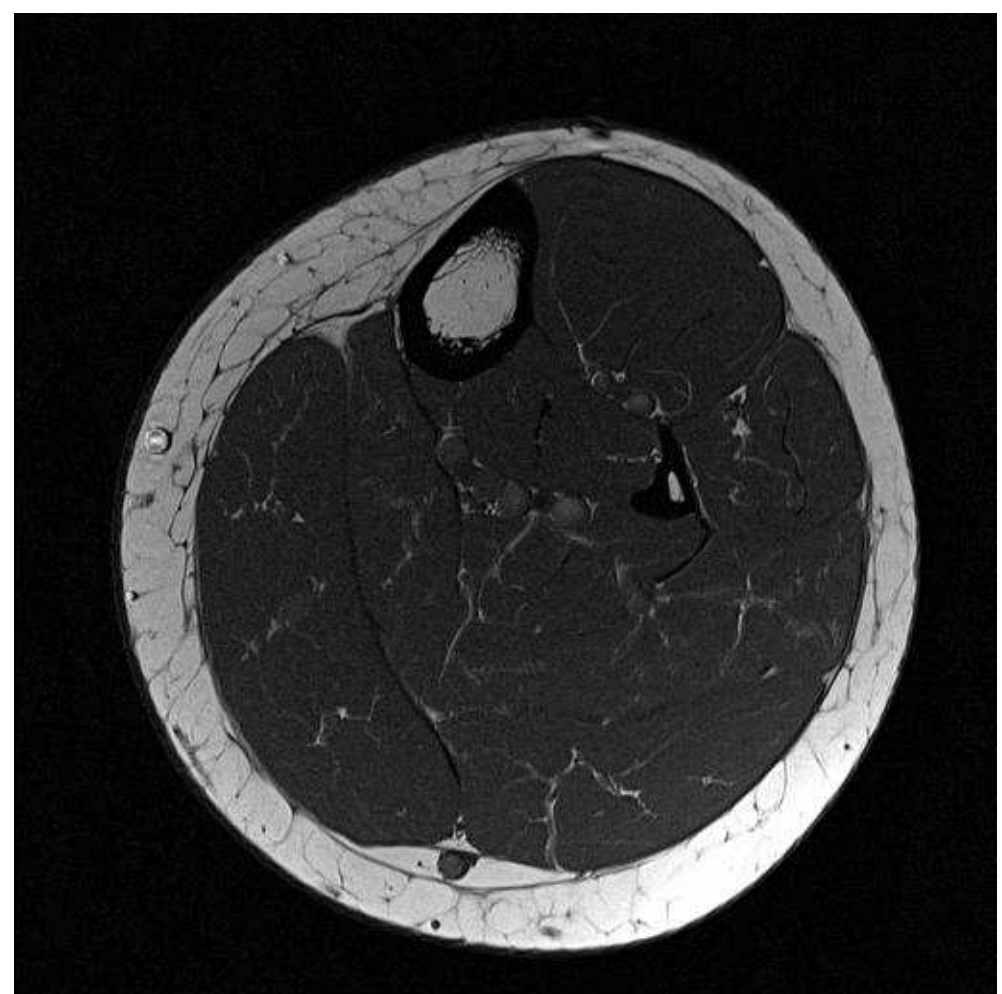

(a)

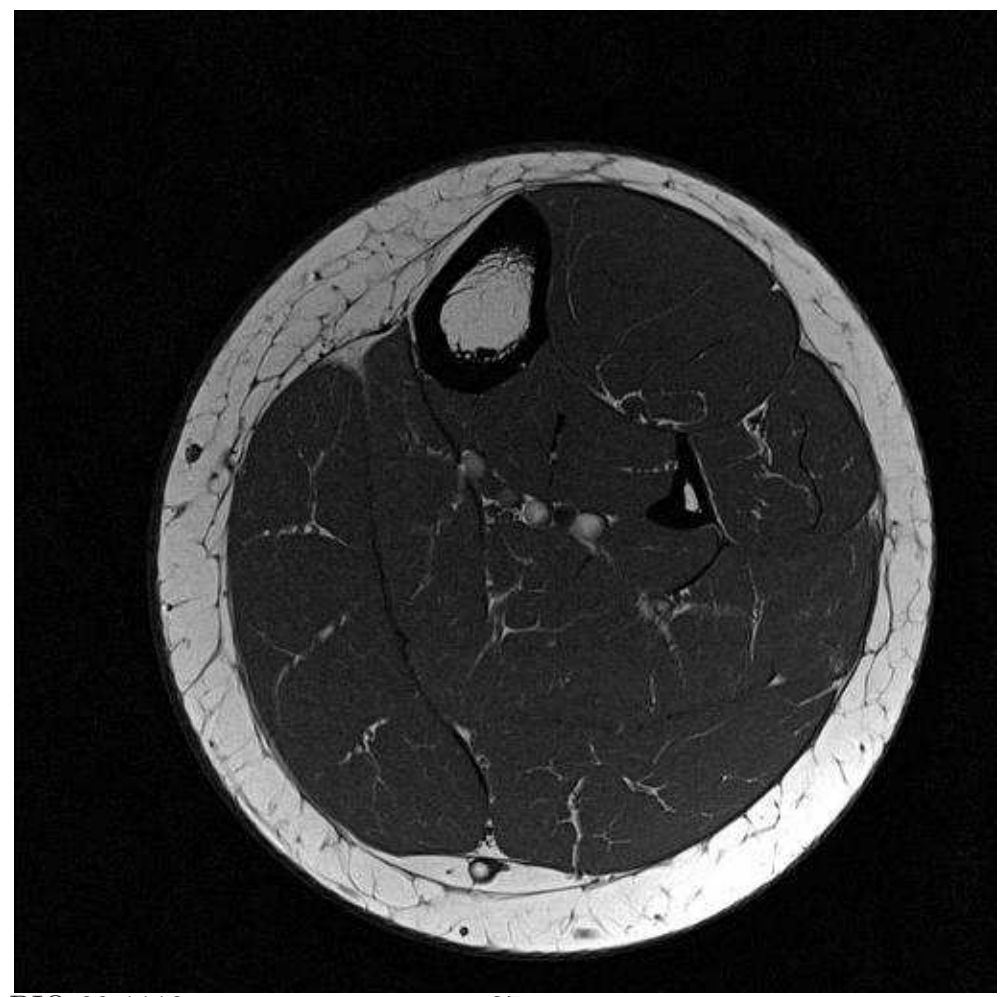

Avril - BIO-09-1119

(3)

Fig. 1 MRI scans of the leg without (a) and with (b) elastic compression garments 


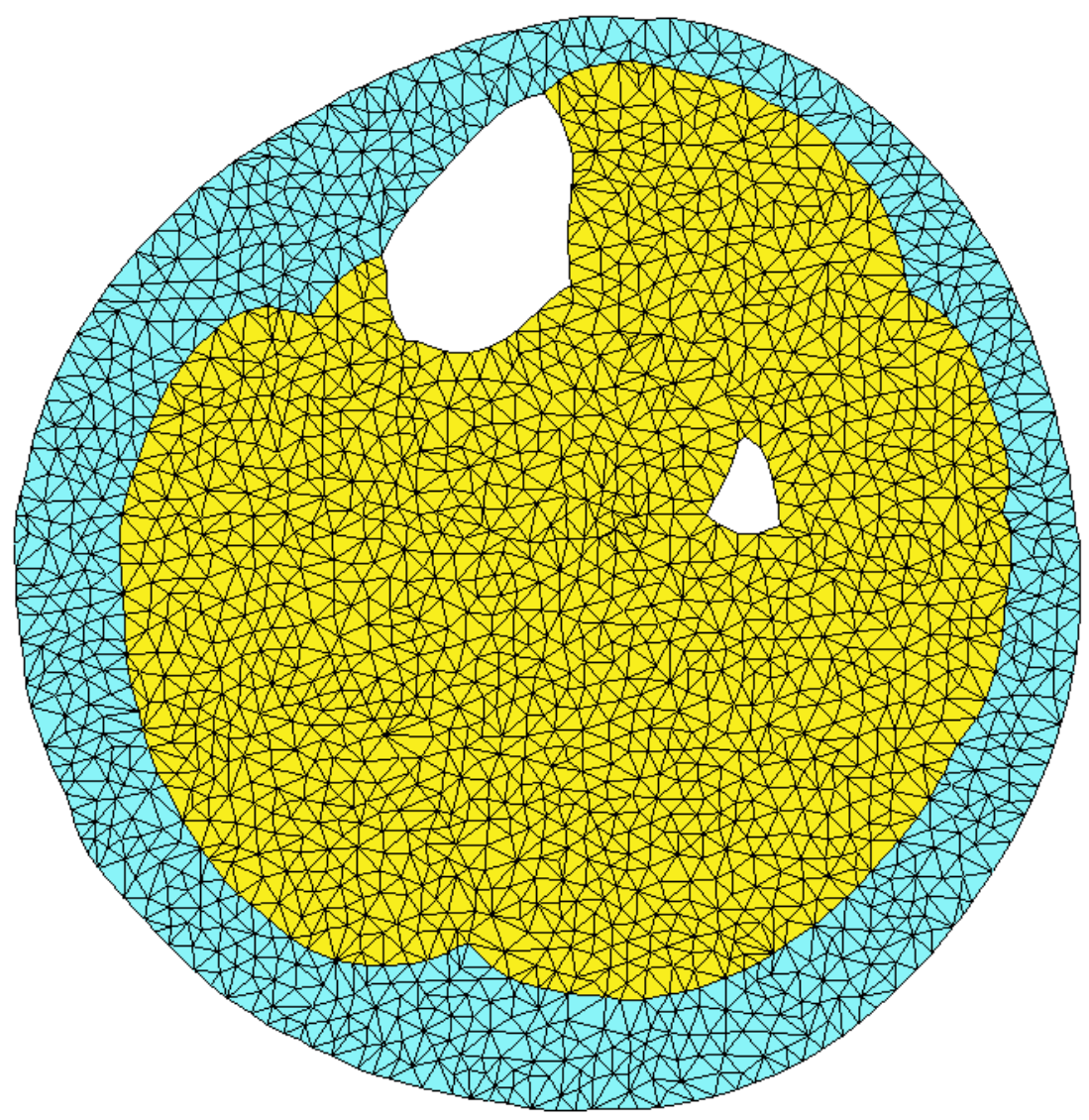

Fig. 2 Mesh of the leg slice in the FE model 


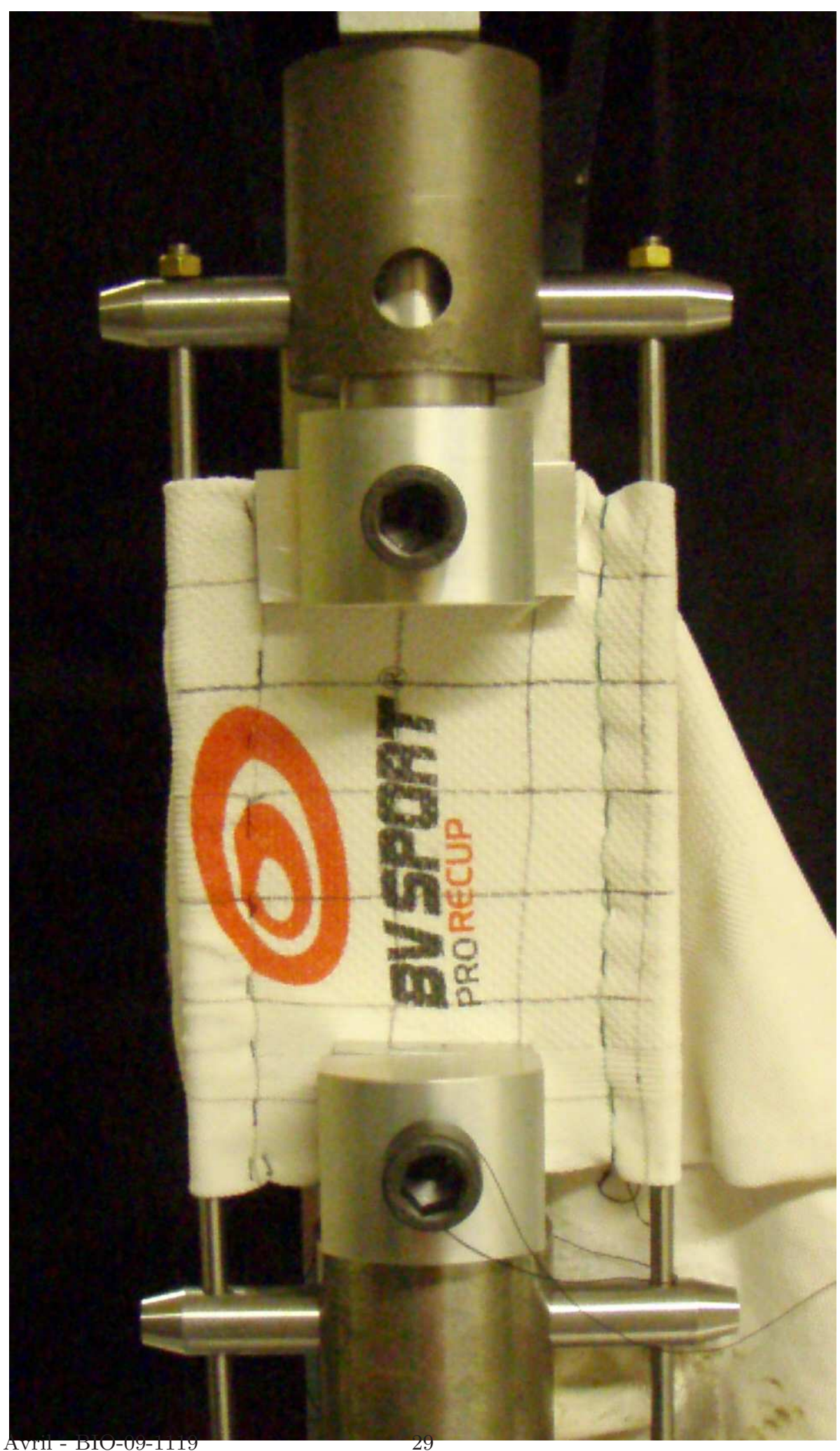

Fig. 3 Set-up used to characterize the elastic behaviour of compression garments 

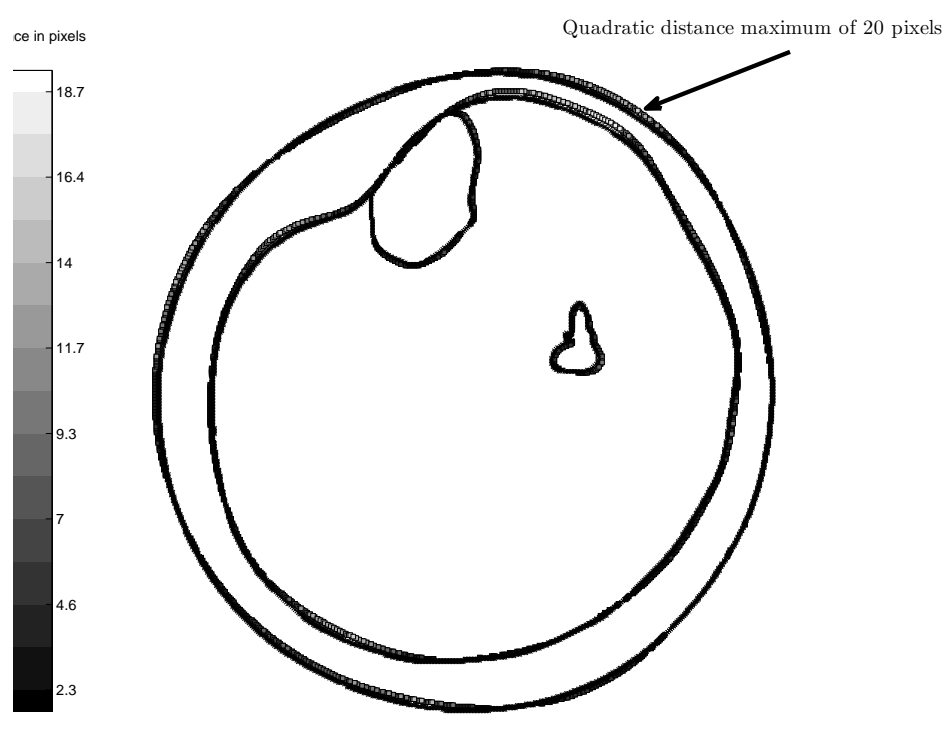

(a)

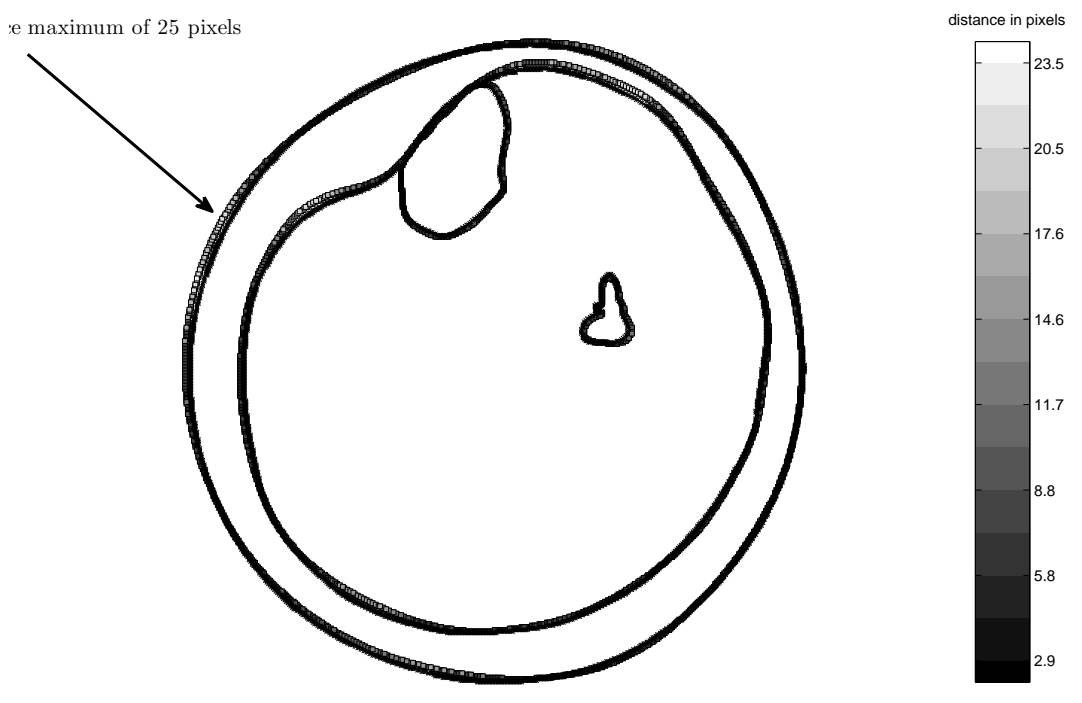

(b)

Fig. 4 Graphical display of the mismatch between the target image and examples of registration, (a) with $\left[C_{10}^{f}, C_{10}^{m}, K_{v}^{f}, K_{v}^{m}\right]=[3.3 \mathrm{kPa}, 11.5 \mathrm{kPa}, 89.6 \mathrm{kPa}, 71.9 \mathrm{kPa}]$, (b) with $\left[C_{10}^{f}, C_{10}^{m}\right.$, $\left.K_{v}^{f}, K_{v}^{m}\right]=[12 \mathrm{kPa}, 11.5 \mathrm{kPa}, 89.6 \mathrm{kPa}, 71.9 \mathrm{kPa}] . C_{10}^{f}$ and $K_{v}^{f}$ are the material constants of fat, $C_{10}^{m}$ and $K_{v}^{m}$ are the material constants of the muscle tissue. $C_{10}$ is the constant corresponding to the contribution of the strain first invariant in the Neo-Hookean strain energy function, $K_{v}$ is the constant corresponding to the contribution of compressibility in the Neo-Hookean strain energy function. 


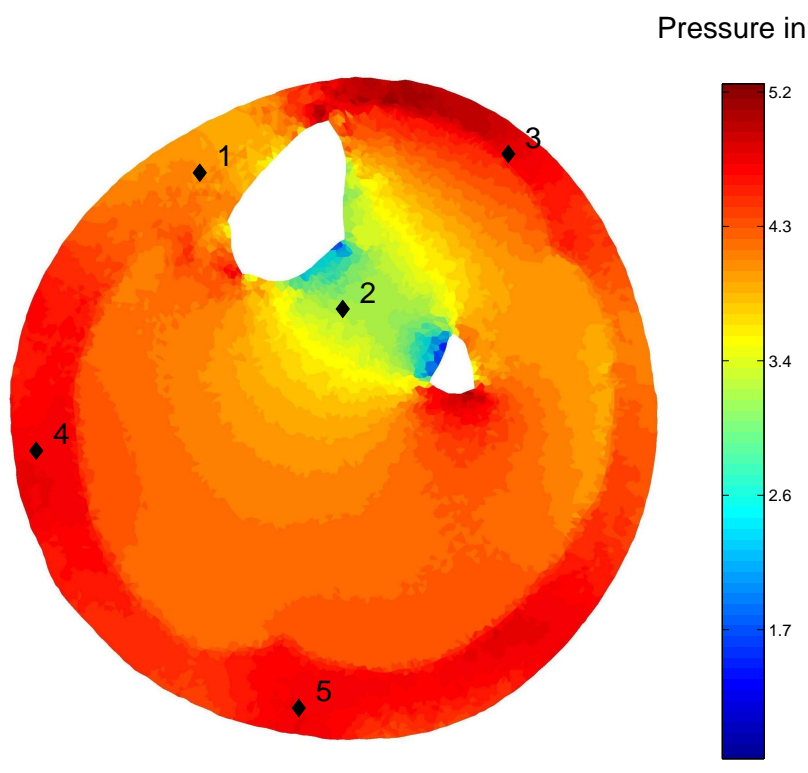

Fig. 5 Hydrostatic pressure computed inside the leg with the central values of elastic properties reported in Tab. 2 


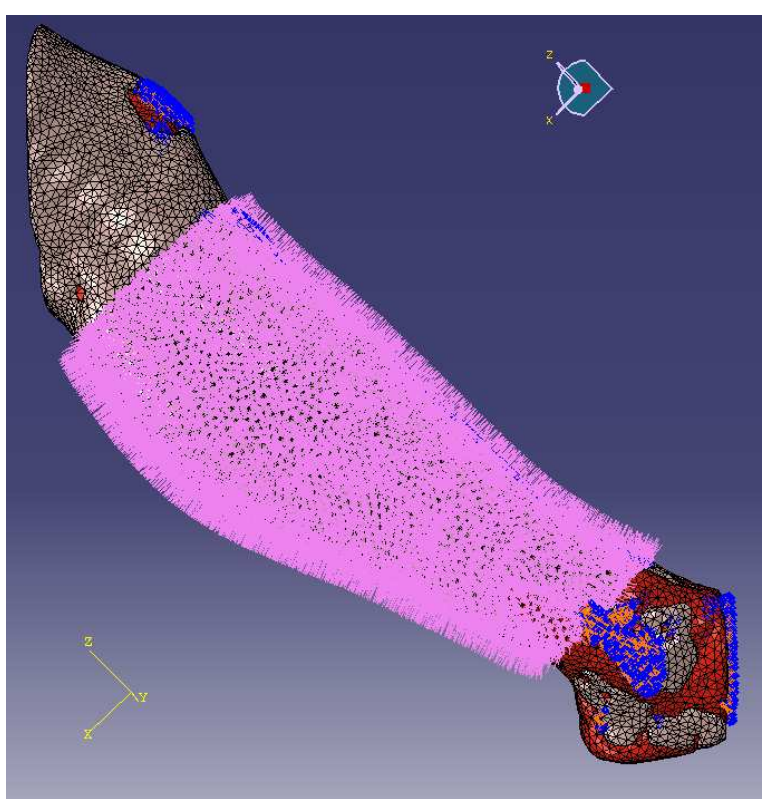

(a)

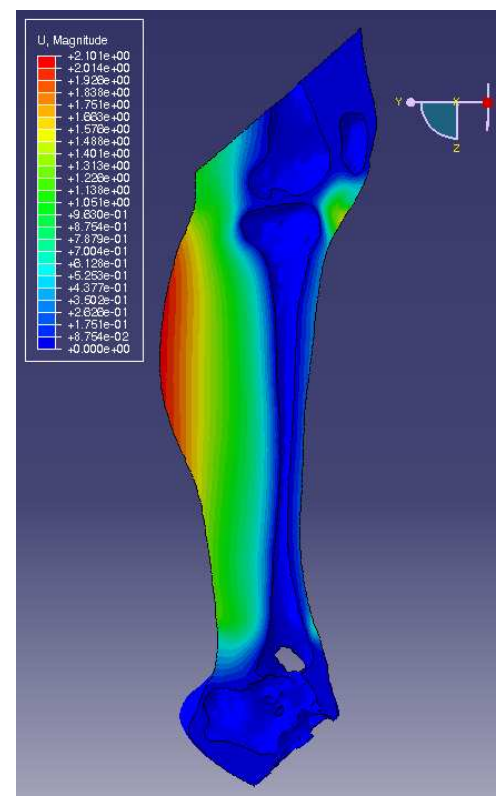

(b)

Fig. 6 3D FE model, (a) mesh and boundary conditions, (b) displacement field in the sagital plane

Avril - BIO-09-1119 